\title{
Out-of-hours discharge from intensive care: certain about uncertainty
}

\author{
Christian Fynbo Christiansen ${ }^{1,2^{*}}$ and Hans Flaatten ${ }^{3,4}$
}

๑ 2018 Springer-Verlag GmbH Germany, part of Springer Nature and ESICM

Discharges out of hours comprise 15\% of discharges from intensive care units (ICUs) to wards [1], and are usually a symptom of strained ICU capacity with demand exceeding the capacity [2]. Premature discharge of patients out of hours may cause insufficient handover and monitoring because the ward or step-down unit often have lower staffing out of hours and may lack necessary equipment for monitoring. Therefore, out-of-hours discharge is a frequently used indicator for ICU quality of care [2].

In an article recently published in Intensive Care Medicine, Vollam and colleagues presented results from a well-conducted systematic review and meta-analysis examining the association between out-of-hours discharge from the ICU and readmission and hospital mortality, respectively [3]. The review and meta-analyses were thoroughly planned and the protocol was previously published [4]. The review included 18 observational studies with a total of 1,191,178 patients and found out-ofhours discharge to be associated with a $30 \%$ increased risk of readmission and 39\% increased risk of in-hospital death.

The current meta-analysis underscores that patients discharged from the ICU out of hours comprise a highrisk group, which is in itself important information. The obvious next question is whether readmission and mortality rate could be reduced by avoiding out-of-hours discharge. This would probably require more ICU beds and any benefit of longer ICU stay should outweigh the increased risk of ICU complications such as nosocomial infections. To answer this question is not easy. Although the current large meta-analysis provides precise estimates of the association, systematic variation from bias

\footnotetext{
*Correspondence: cfc@clin.au.dk

${ }^{1}$ Department of Clinical Epidemiology, Aarhus University Hospital, Olof Palmes Alle 43-45, 8200 Aarhus N, Denmark

Full author information is available at the end of the article
}

and confounding is not reduced with increased sample size. It is therefore crucial to consider other factors associated with both out-of-hours discharge and mortality, i.e., confounding factors [5]. Patients discharged out of hours may differ from patients discharged during the daytime. While there was no major difference in age and sex, there were differences in proportion of surgical patients and severity of illness in the included studies reporting these data [3]. The meta-analysis excluded patients in selected ICU populations although such restriction to a more homogenous population could be an effective way of reducing confounding. The main analysis was not adjusted for confounding because not all included studies controlled for confounding. However, adjustment attenuated the association in studies controlling for confounding. Despite that, the association persisted after adjustment and it should therefore be considered whether there is a true causal association or if any uncontrolled confounding could explain the association. Most studies lack information about reason for admission, severity of disease (organ dysfunction) at discharge, organ-supportive treatment during the ICU stay, and treatment limitations present at ICU discharge; all these are linked to increased mortality (Fig. 1). Treatment limitations is a particularly strong risk factor for death after ICU discharge and half of deaths after ICU discharge is in patients with treatment limitations [6].

It is also difficult to separate planned from unplanned ICU discharge out of hours. Planned discharges during the daytime may be delayed, e.g., due to lack of beds in the ward. Thus, delay of discharge by more than $6 \mathrm{~h}$ was reported in almost half of ICU discharges in Australia and New Zealand, and 34\% of these patients were discharged out of hours [7]. In addition, patients with short-stay observation or treatment in the ICU may be discharged out of hours when intensive care is no longer needed. Finally, patients with treatment limitations may

\section{Springer}




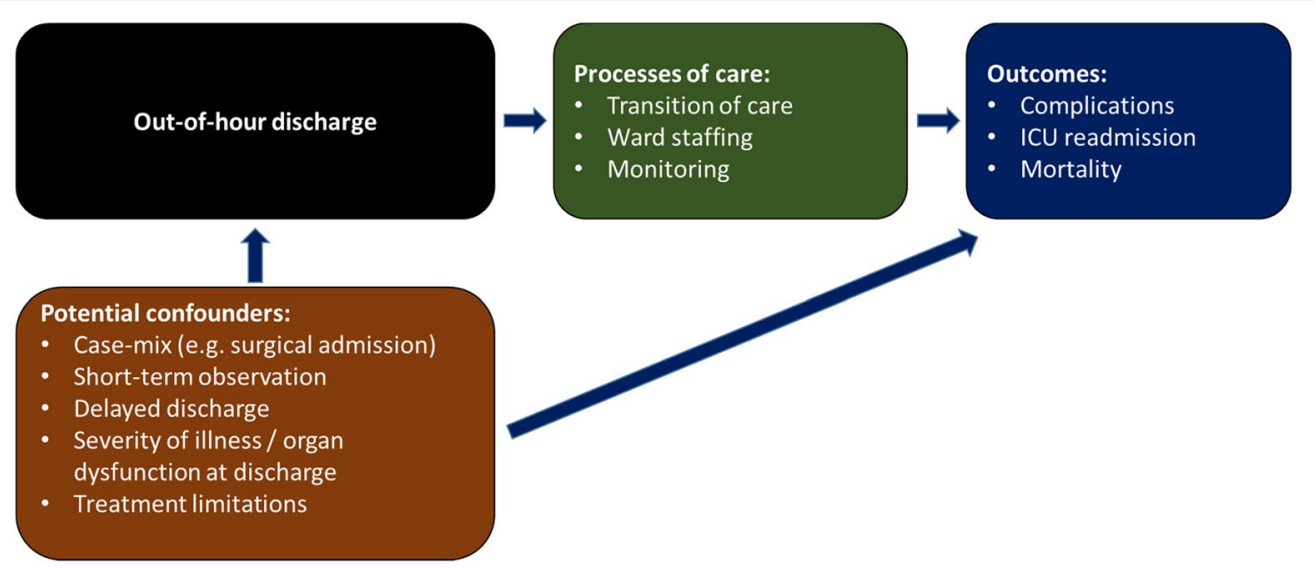

Fig. 1 Potential mechanisms and confounders of the association between out-of-hours discharge and outcomes

be transferred when the patient is no longer a candidate for organ supportive treatment.

The findings by Vollam et al. extend results from a previous review and meta-analysis on time of discharge from ICU and mortality by including studies with and without adjustment for severity of illness and by including ICU readmission as an outcome [1]. The inclusion of readmission as an outcome in the current study is important, because it may be a marker of deterioration after discharge. The previous review and meta-analysis by Yang et al. included 14 observational cohort studies of 953,312 patients [1]. Their finding of increased mortality by $33 \%$ in out-of-hours discharge compared with in-hours discharge is compatible with the current meta-analysis. Restriction to studies that adjusted for severity of illness at ICU admission or discharge had virtually no impact on the results. Patients discharged during the night were more likely to have a treatment-limitation order. Nevertheless, the previous meta-analysis also only found slight attenuation of the association when restricting to studies adjusting for treatment limitations.

The current meta-analysis is important because it emphasizes that patients discharged from the ICU during out of hours comprise a population at particular risk of readmission and death. Both meta-analyses reported in-hospital mortality and lacked data on mortality within a fixed period (e.g., 30-day mortality), which could have reduced the influence of local transferal and discharge patterns $[8,9]$. There is a need for studies addressing the limitations in the current literature, i.e., with improved confounder adjustment and fixed follow-up. There is also a need for more knowledge about processes of care, specific complications, and the reasons for death after out-of-hours discharge that could be targets for future preventive initiatives. Such information is crucial when designing future health care systems including the number of ICU beds and transition of care.
While strong evidence from observational studies confirms that out-of-hours discharge is associated with increased ICU readmission rate and hospital mortality, we need more knowledge to understand why before we can decide how to intervene.

\section{Author details \\ ${ }^{1}$ Department of Clinical Epidemiology, Aarhus University Hospital, Olof Palmes Alle 43-45, 8200 Aarhus N, Denmark. ${ }^{2}$ Department of Clinical Medicine, Aarhus University, Aarhus, Denmark. ${ }^{3}$ Department of Anaesthesia and Inten- sive Care, Haukeland University Hospital, Bergen, Norway. ${ }^{4}$ Department of Clinical Medicine, University of Bergen, Bergen, Norway.}

\section{Compliance with ethical standards}

Conflicts of interest

The authors declare that they have no competing interests.

Received: 6 July 2018 Accepted: 12 July 2018

Published online: 31 July 2018

\section{References}

1. Yang S, Wang Z, Liu Z, Wang J, Ma L (2016) Association between time of discharge from ICU and hospital mortality: a systematic review and metaanalysis. Criti care (Lond Engl) 20:390

2. Rewa OG, Stelfox HT, Ingolfsson A, Zygun DA, Featherstone R, Opgenorth D, Bagshaw SM (2018) Indicators of intensive care unit capacity strain: a systematic review. Crit care (Lond Engl) 22:86

3. Vollam SA, Dutton S, Lamb S, Petrinic T, Young JD, Watkinson P (2018) Out-of-hours discharge from intensive care, in-hospital mortality and intensive care readmission rates: a systematic review and meta-analysis. Intensive Care Med. https://doi.org/10.1007/s00134-018-5245-2

4. Vollam SA, Dutton SJ, Young D, Watkinson PJ (2015) Out-of-hours discharge from intensive care, in-hospital mortality and intensive care readmission rates: a systematic review protocol. Syst Rev 4:93

5. Stroup DF, Berlin JA, Morton SC, Olkin I, Williamson GD, Rennie D, Moher D, Becker BJ, Sipe TA, Thacker SB (2000) Meta-analysis of observational studies in epidemiology: a proposal for reporting. Meta-analysis Of observational studies in epidemiology (MOOSE) group. JAMA 283:2008-2012

6. Santamaria JD, Duke GJ, Pilcher DV, Cooper DJ, Moran J, Bellomo R (2015) The timing of discharge from the intensive care unit and subsequent mortality. A prospective, multicenter study. Am J Respir Crit Care Med 191:1033-1039 
7. Tiruvoipati R, Botha J, Fletcher J, Gangopadhyay H, Majumdar M, Vij S, Paul E, Pilcher D, Australia, New Zealand Intensive Care Society Clinical Trials G (2017) Intensive care discharge delay is associated with increased hospital length of stay: a multicentre prospective observational study. PloS one 12:e0181827

8. Drye EE, Normand SL, Wang Y, Ross JS, Schreiner GC, Han L, Rapp M, Krumholz HM (2012) Comparison of hospital risk-standardized mortality rates calculated by using in-hospital and 30-day models: an observational study with implications for hospital profiling. Ann Intern Med 156:19-26
9. Rydenfelt K, Engerstrom L, Walther S, Sjoberg F, Stromberg U, Samuelsson C (2015) In-hospital vs. 30-day mortality in the critically ill-a 2-year Swedish intensive care cohort analysis. Acta Anaesthesiol Scand 59:846-858 